\title{
The use of research results: at which stage are we in Brazil?
}

\author{
Division of Rheumatology, \\ Escola Paulista de Medicina - São Paulo, Brazil
}

$\mathrm{H}$ ealth research can clearly make an important contribution to development and human wellbeing. The World Bank's 1993 World Development Report presented some convincing examples that investments in health research and development have yielded high returns in better health $(1,2)$. Althought his fact has already been noticed, there is a common agreement, however, that the world's health research programs have only used, so far, a small portion of their potential contribution. In addition to the fact that, apparently, the investment in research in developing country health problems has probably been static or even declining over the past decade (2), there is a critical need also for good quality research. It is hard to believe that

\section{Address for correspondence: \\ Marcos Bosi Ferraz \\ McMaster University - HSC \\ Department of Clinical Epidemiology \& Biostatistics \\ 2C7 - 1200 Main Street West - Hamilton}

Ontario, Canada - L8N $3 Z 5$ much of the research being done, mainly in the developing world, is of adequate scientific quality to provide for valid findings.

Furthermore, most of the high quality research that is performed in these countries, in fact, is of limited relevance with respect to the needs of the poor.

Based on these aspects, there is an urgent need to not only increase quantity, but also the quality and the relevance of health research, particularly in the developing world. Training of personnel and establishment of capable research units are of extreme importance to serve as a resource base for such a type of endeavour. Simultaneously, with the strengthening capacity, it is important to redirect the research to solve issues of importance and improve health conditions.

Once the health research is available, presents a good scientific quality and tackles questions of relevance, the last step to overcome is the implementation of as many of the study results as possible, and the soonest as possible.

The main question to be answered by each country willing to go through this process is related to the definition of the stage at which the country is in this process. It is well-known that for the development and implementation 
of any research, a minimum of structure must be available. If the minimum structure is available, it is important not only to carry through a research project but also to disseminate the concepts related to the design, measurement and evaluation of this research. The point being made here is that a parallel continuous educational structure has to be built in order to allow the expansion of the research initiatives. In this sense, it is important to evaluate and pinpoint the country's stage progress (stage of development). Coming back to the issue of credibility, it is crucial for the researcher who is teaching research methodology (or any other discipline) to be credible among his/her colleagues. The scientific community is structured in a way that values the published research productivity regardless of its relevance and, therefore, it is important for any researcher to be productive; he will attract the attention and respect ability among his colleagues and may, in fact, facilitate his function to spread the knowledge in terms of research empowering. In this sense, in each setting, the research stage must be looked at carefully, and perhaps in some places, an irrelevant but published research may be of as much value as an extremely relevant research. Once the credibility is obtained and the knowledge is spread, it is understandable that all the attention should be focused on the local level priority research. At this stage, the identification of research areas to be tackled should be carefully examined and discussed. In a world of scarce resources, the most suitable strategy should be clearly defined. Certainly, the inter-disciplinary approach at the conception of the research project would increase the chances of the implementation of its results, but the methodology and its scientific validity should never be threatened by any group discussion.

Regarding the dissemination of scientific information, recently, Lomas reviewed the routes through which information and its implications are transmitted from the biomedical research community to practioners who deliver clinical services or their representative organizations (3). Lomas described 3 phases of research information transference into clinical practice: 1. Passive diffusion; 2. Active dissemination; and 3. Coordinated implementation.

Passive diffusion guided the design of research transfer structures until recently, where users of information actively seek after research data from which they can select and appraise information appropriately, and subsequently make research-driven, probable patient care decisions. Based on studies published so far practitioners' behaviour is only loosely connected to formally published research studies $(4,5)$. The continuous growing in the volume of literature certainly posed an obstacle to passive diffusion. Active dissemination emerged then as a solution for the immense amount of scientific data being generated and not fully consumed. Active dissemination is based on the work of groups of people who are ideally credible to accurately synthesize and disseminate information and its implication for clinical practice, in formats such as practice guidelines. The overall validity of the synthesis or its applicability to a particular local environment may be in question. It requires, therefore, the synthesis (and update) of the scientific literature in the most, if not all specialities and sub-specialities, which is understandably complex. In the third and more recently adopted strategy, the coordinated implementation, research information must be carefuliy embedded in multiple routes of influence in order to pressure practitioners for applying it to patient care. In this scenario emerges the overall practice environment, where the administrative, educational, economic and community environments interact with the practitioner forcing the adoption of research findings into local practice.

Finally, the impact of research information depends on its ability to change not only beliefs but also policy assumptions within the relevant audiences(6). It is important to emphasize that raw information is not usable knowledge and a process is needed to transform the research information into knowledge usable in policy practice. Decisionmakers targets have to be identified and reached, and the public has to be directed to a certain level of knowledge. This constitutes the fertile ground on which use of certain types of research information may be made in the future(6).

In conclusion, all strategies should be considered (thought about, weighted, compared) and used to enhance the utilization of research results. It should also be kept in mind that developed and developing countries or even different developing countries face distinct stages in research development and implementation. Research quality and researcher credibility should be established before or at least simultaneously with implementation of any research result; therefore, all the stages to achieve this status should also be considered and respected.

\section{REFERENCES}

1. International Bank for Reconstruction and Development. World Development Report 1993: Investing in Health. New York, Oxford University Press, 1993, p. 168. 
2. Anonymous. Strengthening the contribution of Health Research, Issues, Needs, Options, Questions. Conference Discussion Paper. Conference on Future Partnerships for the Acceleration of Health development. Ottawa, October, 1993.

3. LOMAS, J. - Retailing Research: Increasing the role of Evidence in Clinical services for Childbirth. The Milbank Quarterly 71(3):439-475, 1993.

4. EDDY, D.M. - Clinical Policies and the Quality of Clinical Practice. New England Journal of Medicine 307:343-347, 1982.
5. HAYNES, R.B. - Loose Connections between Peer-Reviewed clinical Journals and Clinical Practice. Annals of Internal Medicine 113:724-7, 1990.

6. LOMAS, J, - Finding Audiences, Changing Beliefs: The Structure of Research Use in Canadian Health Policy. J Health Politics, Policy and Law 15(3):525-542, 1990. 